\title{
MODELLING METHODS OF MAGNETOHYDRODYNAMIC PHENOMENA OCCURRING IN A CHANNEL OF THE DEVICE USED TO WASH OUT THE SPENT AUTOMOTIVE CATALYST BY A LIQUID METAL
}

\begin{abstract}
The recovery of precious metals is necessary for environmental and economic reasons. Spent catalysts from automotive industry containing precious metals are very attractive recyclable material as the devices have to be periodically renovated and eventually replaced. This paper presents the method of removing platinum from the spent catalytic converters applying lead as a collector metal in a device used to wash out by using mangetohydrodynamic stirrer. The article includes the description of the methods used for modeling of magnetohydrodynamic phenomena (coupled analysis of the electromagnetic, temperature and flow fields) occurring in this particular device. The paper describes the general phenomena and ways of coupling the various physical fields for this type of calculation. The basic computational techniques with a discussion of their advantages and disadvantages are presented.
\end{abstract}

Keywords: magnetohydrodynamic, precious metals wash out, automotive spent catalyst

\section{Introduction}

The auto catalytic converter consists of steel or ceramic shell containing the carrier of honeycomb structure covered by the Precious Group Metals (PGM), especially platinum, palladium and rhodium. Such construction increases the active surface, namely the contact area of catalytic substances (Pt, Pd, $\mathrm{Rh}$ ) with exhaust gases which flow through the channels. TWC (Three-Way Catalyst) is a modern catalytic converter in which three reactions take place simultaneousely: two oxidation reactions of $\mathrm{CO}$ to $\mathrm{CO}_{2}$ and $\mathrm{HC}$ to $\mathrm{H}_{2} \mathrm{O}$, and reduction of $\mathrm{NO}_{\mathrm{X}}$ to $\mathrm{N}_{2}$. As a result, the substances appearing at the outlet of the converter, like carbon dioxide, water and nitrogen, are neutral to the environment [1].

Spent catalytic converters containing precious metals are very attractive recyclable material as the devices have to be periodically renovated and eventually replaced. Used converters can be processed pyro- and hydrometalurgically; mixed methods are also adopted [2]. The process of recovery of both precious and other metals is extremely beneficial because as a result it reduces the amount of waste that needs to be disposed of conventionally, saves natural resources and reduces energy usage $[3,4]$. However, during the process of recovery of these attractive metals some difficulties are encountered. A single catalytic converter contains at most a few grams of platinum metals and they are captured in the capillary structure of its usually ceramic carrier. For this reason, in order to make the whole procedure economic, the cost of processing a single converter should be maximally reduced.
This paper presents a possible way of removing platinum from the spent catalytic converters applying lead as a collector metal in a device used to wash out waste by using mangetohydrodynamic stirrer. The magneto-hydrometallurgical device forced circulation of liquid metal under the influence of electromagnetic fields. The movement of the metal collector speeds up leaching of precious metals. Use of a closed cycle increases the concentration of precious metals in the metal collectors [5]. The article includes the description of the methods used for modeling of magnetohydrodynamic phenomena (coupled analysis of the electromagnetic temperature and flow) occurring in this particular device [6]. The general phenomena and ways of coupling the various physical fields for this type of device were also presented.

\section{Mathematical description of physical fields analysed in the model of a device for washing out catalytic converters with a liquid metal}

Numerical modelling of the phenomena occurring in the process of washing the converters with a liquid metal whose movement is forced by the electromagnetic field requires that the analyses of a least three physical fields must be considered: the electromagnetic field, temperature field and flow field. Mutual dependences of the three is presented in Fig. 1. Modelling of each of these fields is a serious research challenge, which is further complicated by the couplings that take place among them.

\footnotetext{
* SILESIAN UNIVERSITY OF TECHNOLOGY, INSTITUTE OF METALS TECHNOLOGY, 8 KRASINSKIEGO STR., 40-019 KATOWICE,POLAND

** SILESIAN UNIVERSITY OF TECHNOLOGY, DEPARTMENT OF INDUSTRIAL INFORMATICS, 8 KRASINSKIEGO STR., 40-019 KATOWICE,POLAND

\# Corresponding author: Agnieszka.Fornalczyk@polsl.pl
} 


$$
\begin{aligned}
& \nabla \times \boldsymbol{E}=-\frac{\partial \boldsymbol{B}}{\partial t} \\
& \nabla \times \boldsymbol{H}=\boldsymbol{J}
\end{aligned}
$$

complemented with constitutive equations (5), (6), (7):

$$
\begin{aligned}
\mathbf{D} & =e \mathbf{E} \\
\boldsymbol{B} & =\mu \boldsymbol{H} \\
\boldsymbol{J} & =\gamma \boldsymbol{E}
\end{aligned}
$$

Fig. 1. Dependence of physical fields in modelling of magnetohydrodynamic phenomena; $v$ - velocity, $\mathrm{f}$ - volumetric density of electromagnetic forces, $\eta$ - viscosity, $\rho$ - density, $q-$ volumetric density of heat sources, $\sigma-$ conductivity

Numerical model describing an electromagnetically forced flow of the liquid metal through the capillary structure of the converter may have various forms. The most significant differences among the models will concern the number of the geometric dimensions considered in the model, the number of calculation areas, uniformity of calculation grids, and the domain in which the electromagnetic field is analysed.

Mathematical description and computational model for the analysis of the electromagnetic field

The analysis of the electromagnetic field is always based on Maxwell's equations in the following form $[7,8]$ :

$$
\begin{gathered}
\nabla \cdot \boldsymbol{D}=\rho_{v} \\
\nabla \cdot \boldsymbol{B}=0
\end{gathered}
$$

where:

D - electric induction,

$\rho_{v}$ - electric charge density,

$\boldsymbol{B}$ - magnetic induction,

$\boldsymbol{E}$ - electric field density,

$\boldsymbol{H}$ - magnetic field density,

$\boldsymbol{J}$ - current density,

$e$ - electric permittivity,

$\mu$ - magnetic permeability,

$\gamma$ - conductivity.

The total current density components from Equation (4) are represented by Eq. (8) [6]:

$$
\boldsymbol{J}=\gamma \boldsymbol{E}+\frac{\partial \boldsymbol{D}}{\partial t}+\gamma(\boldsymbol{v} \times \boldsymbol{B})+\boldsymbol{J}_{o b}+\rho \boldsymbol{v}_{k}+\nabla \times(\boldsymbol{D} \times \boldsymbol{v})
$$

where:

$\gamma \boldsymbol{E}$ - transformation current density ( $a$ term of the right side of the eq. 8),

$\frac{\partial \boldsymbol{D}}{\partial t}$ - displacement current density ( $b$ term of the right side of the eq. 8),

$\gamma(\boldsymbol{v} \times \boldsymbol{B})-$ current density induced due to liquide metal

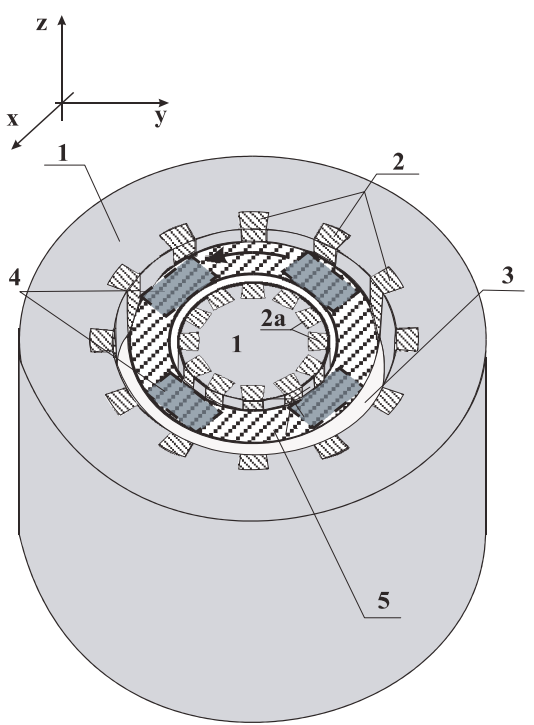

Fig. 2. Scheme of the device for precious metal recovery from spent automotive catalytic converters
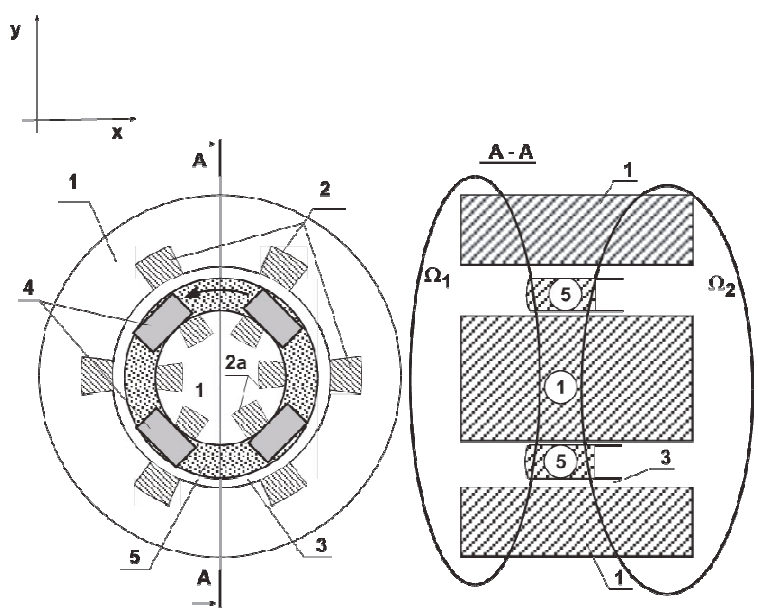

Fig. 3. Cross section of the device for precious metals recover 1 - core, 2 - coils, 3 - air, 4 - converters, 5 - liquid metal 
movement ( $c$ term of the right side of the eq. 8),

$\boldsymbol{J}_{o b}$ - source current density ( $d$ term of the right side of the eq. 8), $\rho v_{k}$ - free particles movement current density ( $e$ term of the right side of the eq. 8),

$\nabla \times(\boldsymbol{D} \times \boldsymbol{v})-$ current density iduced by electric field movement ( $f$ term of the right side of the eq. 8 ).

For the case of modelling the conducting area, in Eq. (1) it is assumed that there are no free charges, while in Eq.

(8) there is no component resulting from the charge flow. (Additionally for low and medium frequencies displacement current is neglected. Since the metal in the electromagnetic field moves in relation to the coils generating the field, elements $c$ ) and $f$ ) should remain in the equation. Still, in the case when the metal movement is much slower than the field variation, these elements can also be neglected. Even after those simplifications, the equations are still difficult to solve because of their mutual couplings.

The equations are further modified in order to express field values by means of just one value, e.g. $\boldsymbol{H}$. After the elimination of $\boldsymbol{E}$, Eq. (4) takes the form of (9) under the assumption that $\mu$ $=$ var, e $=$ const, $\gamma=$ var i $\frac{\partial \mu}{\partial t}=0$.

$$
\nabla^{2} H=\gamma \mu \frac{\partial \mathbf{H}}{\partial t}-\nabla\left(\mathbf{H} \cdot \frac{\nabla \mu}{\mu}\right)-\frac{\nabla \gamma}{\gamma} \times \nabla \times \mathbf{H}-e \nabla \times \frac{\partial \mathbf{E}}{\partial t}
$$

Assuming that the material parameters $\mathrm{e}=$ const and $\gamma=$ const are homogenous, and $\mu=$ var, Eq. (9) takes the following form (10) [8]:

$$
\nabla^{2} H=\gamma \mu \frac{\partial \mathbf{H}}{\partial t}-\nabla\left(\mathbf{H} \cdot \frac{\nabla \mu}{\mu}\right)+\mu e \frac{\partial^{2} \mathbf{H}}{\partial t^{2}}
$$

Another way to simplify the analysis is to use the electrodynamic potentials. The most often used are magnetic vector potential $\boldsymbol{A}$, electric scalar potential $V_{e}$, electric vector potential $\boldsymbol{T}$, magnetic scalar potential $V_{m}$. Making use of the potentials may lead to a smaller number of variables or may unify the boundary conditions .

On the basis of the sourcelessness of the magnetic field(2) and vector calculus identity $\nabla \cdot \nabla \times \boldsymbol{A} \equiv 0$, a notion of magnetic vector potential $\boldsymbol{A}$ (11) is introduced. Based on Kirchhoff's first law in the form of $\nabla \cdot \boldsymbol{J}=0$ and a vector identity identical as in the previous case, a notion of electric vector potential $\boldsymbol{T}$ (12) is introduced $[9,10]$.

$$
\begin{aligned}
& \boldsymbol{B}=\nabla \times \boldsymbol{A} \\
& \boldsymbol{J}=\nabla \times \boldsymbol{T}
\end{aligned}
$$

where:

$\boldsymbol{A}$ - magnetic vector potential,

$\boldsymbol{T}$ - electric vector potential.

$\boldsymbol{B}$ i $\boldsymbol{J}$ are determined from the electrodynamic potentials with the use of rotation, and from vector calculus identity $\nabla \times \nabla V \equiv 0$ it is concluded that gradient rotation is zero, which means that the potentials $\boldsymbol{A}$ i $\boldsymbol{T}$ are determined with accuracy to scalar potential gradient. In order to avoid ambiguity of $\boldsymbol{A}$ or
$\boldsymbol{T}, \nabla \cdot \boldsymbol{A}$ i $\nabla \cdot \boldsymbol{T}$ must be adequately adopted.

To obtain a description of the electromagnetic field using vector potential $\boldsymbol{A}$, Eq. (6) and (11) should be substituted into Equation (4), which results in the following equation:

$$
\nabla \times\left(\frac{1}{\mu} \nabla \times \boldsymbol{A}\right)=\gamma \boldsymbol{E}
$$

Additionally, by substituting vector potential expression (11) into Eq. (3), assuming that displacement currents can be neglected, and making use of vector identities $\nabla \times \nabla V \equiv 0$, the expression for electric field density can be obtained, taking the following form:

$$
\boldsymbol{E}=-\frac{\partial A}{\partial t}-\nabla V_{e}
$$

where:

$V_{e}-$ scalar vector of electric field

Finally after substituting Eq. (13) to Eq. (14) the following expression is obtained:

$$
\nabla \times\left(\frac{1}{\mu} \nabla \times \boldsymbol{A}\right)=\gamma\left(-\frac{\partial A}{\partial t}-\nabla V_{e}\right)
$$

Making use of constitutive dependence (5) and expression for vector potential in Eq. (1), and assuming that no free charges are present, the following equation can be obtained:

$$
\nabla \cdot\left(\gamma \frac{\partial A}{\partial t}+\gamma \nabla V_{e}\right)=0
$$

Since vector potential is expressed with accuracy to scalar field potential, it is additionally defined by calibration. Coulomb and Lorenz calibration are the most commonly used. After applying Coulomb calibration in the form of $\nabla \cdot \boldsymbol{A}=0$, Eq. (15) and (16) take the following form:

$$
\nabla^{2} \boldsymbol{A}=\gamma \mu \frac{\partial \boldsymbol{A}}{\partial t}+\gamma \mu \nabla V_{e}
$$

$$
\nabla \cdot \nabla V_{e}=0
$$

In this way a system of two equations is obtained. Applying Lorentz calibration, separation of the potentials is achieved.

The transformations presented above concern the description of the electromagnetic field in the time domain. This description is the most accurate and allows for the socalled transient states; with this description it is relatively simple to allow for a potential nonlinear magnetic elements (ferromagnetic materials reaching saturation). However, modelling electromagnetic field in the time domain requires heavy calculations because time step must be adjusted to the field change rate. In order to reduce the computation time of the field analysis, it can be run as a symbolic analysis, that is one assuming a sinusoidal of the state variables. This makes it quite difficult to allow for a nonlinear dependence $B=f(H)$ and does not allow determination of transient states. Eq. 17 for 
symbolic notation takes the following form:

$$
\nabla^{2} \boldsymbol{A}=\gamma \mu \mathrm{j} \omega \boldsymbol{A}+\gamma \mu \nabla V_{e}
$$

The ultimate aim of the analysis of the electromagnetic field is determination of volume densities of the electrodynamic forces fe (20) and heat sources qv (21) [8].

$$
\begin{gathered}
\boldsymbol{f}_{e}=\boldsymbol{J} \times \boldsymbol{B} \\
q_{v}=\frac{\boldsymbol{J}^{2}}{\sigma}
\end{gathered}
$$

In the electromagnetic field modeling, it is necessary to allow for all elements of the system which are namely: liquid metal, catalytic converters, coils, magnetic core, and adequate part of the system surroundings. Calculation grid density is dependent on the depth of the electromagnetic field penetration (the smaller the depth, the greater grid density required).

\section{Mathematical description and computational model for an analysis of temperature field}

The analysis of the temperature field may be performed with the use of energy balance equation (22) with adequate boundary and input conditions [9]:

$$
\begin{gathered}
\frac{\partial(\rho W)}{\partial t}+\nabla \cdot(\boldsymbol{v}(\rho W+p))=\nabla \cdot\left(\lambda_{e f} \nabla T+\overline{\overline{\tau_{e f}}} \cdot \boldsymbol{v}\right)+q_{v} \\
\frac{\partial}{\partial t}(\rho h)+\nabla \cdot(\boldsymbol{v} \rho h)=\nabla \cdot\left(\lambda_{e f} \nabla T\right)+q_{J} \\
W=h-\frac{p}{\rho}+\frac{\boldsymbol{v}^{2}}{2}
\end{gathered}
$$

where:

$\rho-$ density

$W-$ specifc energy

$v$ - velocity

$\lambda_{e f}$ - effective thermal conductivity

$T$ - temperature

$q_{v}$ - heat source density

$\overline{\tau_{e f}}-$ effective stress tensor

$h+$ specific sensible ethalpy

$t$ - time;

Eq. (22) describes propagation of energy in gaseous and liquid media. The equation is slightly simplified for incompressible liquids as a eq.(23). The computational model may comprise just the liquid metal or the whole device. In the latter case the model is particularly complex as it must allow for heat release and dissipation in solids, liquids and gases. Calculation mesh for this model depends on the predicted velocities of the media and temperature gradients. The grid must be finer in the areas of intense movement and high temperature gradients.
One of the input conditions is volumetric density of heat sources $\left(q_{v}\right)$ determined in the analysis of the electromagnetic field. As the basic area of interest is the liquid metal, it was decided that the calculations of the temperature field would be limited to this part of the model. Such reduction requires that the conditions of heat release must be specified at the chosen boundaries of the area. They can be modelled in a typical manner with Eq. (25).

$$
-\lambda_{e f}{\frac{\partial T}{\partial n_{P}}}_{P}=\alpha\left(T-T_{o}\right)+c \sigma\left(T^{4}-T_{o}^{4}\right)
$$

where:

$\alpha$-convective heat transfer coefficient

$c$ - emissivity

$\sigma$ - Stefan-Boltzmann constant

$T_{o}$ - ambient temperature;

In this case the difficulty is the right selection of convective heat transfer coefficient and emissivity. In the analysis performed, the temperature field was calculated extremely rarely because the amount of the heat released as a result of electromagnetic activity was scarce and the whole system had to be resistance heated. Owing to this, resistance heating system thermal balance of the system could be obtained (temperature stabilization system).

\section{Mathematical description and computational model for the analysis of the flow field}

Similarly to the temperature field, the calculation area for the flow field may comprise the whole device or just the liquid metal. For the whole device the flow is modelled for two media. First one is air (compressible fluid), which surrounds the whole unit and cools the coils. The other medium for which the flow must be modelled is liquid metal (incompressible fluid). The analysis of the flow field is performed with the use of a system of equations in the form (26), (27), and energy balance Eq. (22) [8,11]:

$$
\begin{gathered}
\frac{\partial \rho}{\partial t}+\nabla \cdot(\rho \boldsymbol{v})=0 \\
\frac{\partial(\rho \boldsymbol{v})}{\partial t}+\nabla \cdot(\rho \boldsymbol{v} \boldsymbol{v})=-\nabla p+\nabla \cdot \overline{\overline{\tau_{e f}}}+\rho \mathrm{g}+\boldsymbol{f}_{e} \\
\overline{\overline{\tau_{e f}}}=\cdot \eta_{e f}\left(\nabla \boldsymbol{v}+\nabla \boldsymbol{v}^{T}\right)-\eta_{e f} \frac{2}{3} \nabla \cdot \boldsymbol{v} I \\
\nabla \cdot \mathbf{v}=0 \\
\rho_{f}\left(\frac{\partial \boldsymbol{v}}{\partial t}+\boldsymbol{v} \cdot \nabla \boldsymbol{v}\right)=-\nabla p+\eta_{e f} \nabla^{2} \boldsymbol{v}+\rho \mathrm{g}+\boldsymbol{f}_{\boldsymbol{e}}
\end{gathered}
$$

where:

$\rho$ - fluid density,

$\boldsymbol{v}$ - velocity vector,

$p$-pressure, 
$\overline{\bar{\tau}}-$ effective stress tensor

$\boldsymbol{g}$ - gravitational acceleration,

$\boldsymbol{f}_{\boldsymbol{e}}$ - electromagnetic force density

$\eta_{f}$ - effective viscosity,

$I$ - unit tensor,

The first equation that was used (26) was the equation of mass conservation, referred to as continuity equation, the second one was the equation expressing the law of conservation of momentum (27). The Eqs (29) and (30) are slightly simplified for incompressible liquids. Modelling the flow is quite a complex issue, one of the problems being modelling the systems with turbulent flows. In order to perform such a direct analysis based on Equations (26), (27) and (22), using for example Direct Numerical Simulation method, very fine grids must be used (the number of nodes proportional to $\mathrm{Re}^{9 / 4}$ ). In order to avoid it, one can introduce a turbulence model. The classic turbulence modelling is based on the Reynold's assumption that in the case of a turbulent flow all the parameters that describe the flow can be expressed by the sum of two components: average value and turbulent fluctuation. An introduction of the values described in this way to Navier-Stokes equations leads to the appearance of new surface forces - turbulent stresses. In the mathematical description of the problem an additional value appears, which makes the equation system underdetermined. In order to reduce the number of variables and resolve this problem, an adequate model of turbulence should be introduced. A model used quite frequently is a two-equation one, $k-\varepsilon$, introducing two additional equations: transport equation of turbulence kinetic energy $k$ and transport equation of turbulence kinetic energy dissipation rate $\varepsilon$. These equations require an introduction of additional empirical coefficients, but the equation system describing fluid movement is no longer underdetermined. Effective viscosity can be determined from $k-\varepsilon$ model by substituting Eq.(32) into Eq. (31):

$$
\begin{gathered}
\eta_{e f}=\eta+\eta_{t} \\
\eta_{t}=\rho C \frac{k^{2}}{\varepsilon}
\end{gathered}
$$

where:

$\eta_{e f}-$ effective viscosity,

$\eta_{t}-$ turbulent viscosity,

$k$-kinetic energy of turbulence,

$\varepsilon$ - energy dissipation rate of turbulence,

$C$ - empirical constant.

The principal advantage of model $k-\varepsilon$ is that it yields very good results for many realistic flows that are technically relevant [12].

Another problem with flow modelling is how to allow for the structures with very small geometric dimensions. Considering them as they are will require a still finer calculation grid.

In the case considered it was assumed that the flow field would be determined only for the liquid metal, partly because the temperature of the whole system was stabilized and thus heat release conditions were of no significance. In order to further simplify the calculations of the flow field, the part of channel occupied by converters were modelled as anisotropic porous medium with laminar flow and pressure drop consistent with Hagen-Poiseuille's equation:

$$
\frac{\Delta p}{L}=\frac{28.5}{d^{2}} \eta v
$$

where:

$\Delta \mathrm{p}$ - pressure drop along capillary length $\mathrm{L}$, $d$-hydraulic diameter of the capillary,

$v$ - flow velocity

$\eta$-dynamic viscosity.

A module $f_{R}$ representing porous medium resistance (34) appears within the converter area in Navier-Stokes equation (30), while a module of electromagnetic field activity $\boldsymbol{f}_{e}$ disappears since the macroscopic conductivity of the area in the direction of the current flow is zero[8].

$$
\boldsymbol{f}_{R}=-\frac{28.5}{d^{2}} \eta(\boldsymbol{n} \cdot \boldsymbol{v}) \boldsymbol{n}
$$

where:

$\mathbf{n}$ - unit vector of a capillary direction.

\section{Summary}

For the sake of numerical modelling of the device for washing out the catalytic converters with a liquid metal, the models selected for calculations differed in the way the fields were coupled and in the density of the discretization meshes. It was decided that a symbolic analysis of the electromagnetic field analysis would be performed and the grids for this analyses of the electromagnetic field, flow field and temperature field would be different. The values of volumetric density of the electromagnetic field, determined in the analysis of the electromagnetic field were calculated as average for time period and as such they were introduced into the flow field calculations. It was also assumed that metal movement does not cause induction of any additional current (component $\mathrm{c}$ and $f$ in Eq. (8) are zero). This simplification can be accepted because metal is much lower than the electromagnetic field change rate $[13,14]$.

Reanalysis of the electromagnetic field is performed only when the shape of the metal surface changes (if the analysis of the meniscus shape is performed) or in the case of significant temperature changes. Because of the complexity of the computational model, it was decided that the number of geometric dimensions would be limited to just two of them.

The advantages of the above model:

1. Limiting the number of geometric dimensions means evident simplification of the model, but at the same time leads to a serious simplification of the model for the analysis of the electromagnetic field because for twodimensional systems mathematical description changes from a vector one to scalar one (for models with magnetic vector potential).

2. The analysis of the electromagnetic field in the symbolic domain makes it possible to perform the calculations of 
the distribution of the electromagnetic field only once in the case when a change in temperature does not occur (in the case considered the system is thermally stabilized), and the analysis of the liquid meniscus is skipped.

3. The separation of the calculation grids for the model for the electromagnetic calculations and for the flow filed saves the calculation time significantly.

However, there are also some drawbacks of the model:

1. When considering only two geometric dimensions, it is not possible to allow for the phenomena occurring in the areas marked as $\Omega_{1}, \Omega_{2}$ in Fig. 3 .

2. The separation of the calculation grids requires an algorithm for an interpolation of the results obtained in the analysis of the electromagnetic field, so that the values $\boldsymbol{f}_{v} \mathrm{i} q_{v}$ (determined in the EM analysis) could be put assigned to [15] all the nodes of the calculation mesh for the model for the analysis of the flow field.

3. Symbolic modelling of the electromagnetic field complicates the issue of allowing for the nonlinear characteristics $B=f(H)$ in the calculations.

\section{REFERENCES}

[1] M. Saternus, A. Fornalczyk, Metalurgija Metallurg 50 4, 261 $264(2011)$

[2] J.S. Yoo, Catalyst Today 44, 27-46, (1998)
[3] B. Oleksiak, G. Siwiec, A. Blacha-Grzechnik, J. Wieczorek, Metalurgija Metallurgy 53 4, 605-608 (2014)

[4] B. Oleksiak, G. Siwiec, A. Blacha-Grzechik, Metalurgija Metallurgy 52 1, 107-110 (2013)

[5] http://www.ztch.umcs.lublin.pl/materialy/rozdzial_26.pdf, 2015.01.08

[6] A. Fornalczyk, S. Golak, M. Saternus, Mathematical Problems in Engineering, ID 461085 doi:10.1155/2013/461085 (2013)

[7] J. Hauser, Elektrotechnika - Podstawy elektrotermii i techniki świetlnej. Wydawnictwo Politechniki Poznańskiej, Poznań 2006.

[8] M. Hering, Podstawy elektrotermii. 2. WNT, Warszawa 1992.

[9] http://orange.engr.ucdavis.edu/Documentation 12.1/, 2015.01.15.

[10] A. Krawczyk, J. Tegopoulos, Numerical modeling of eddy currents. Clarendon Press, Oxford 1993.

[11] T. Nakata, N. Takahashi, K. Fujiwara, K. Muramatsu, Z. Cheng, IEEE Transaction on Magnetics, 24 6, 3159 - 3161 (1988)

[12] D. Wilcox, Turbulence modeling for CFD, 3rd ed. DCW Industries 2006

[13] T. Merder, Archives of Metallurgy and Materials 58, 4,11111117 (2013)

[14] T. Merder. J. Pieprzyca, M. Saternus, Metalurgia 53, 2, 155 157 (2014)

[15] A. Smalcerz, R. Przylucki, International Journal of Thermophysics 34, 4, 667-679 (2013) 